\title{
RETRACTED ARTICLE: Computer electromagnetic radiation carcinogenic doses based on Monte Carlo algorithm
}

\author{
Haoli Hou ${ }^{1} \cdot$ Junhua Wang ${ }^{1} \cdot$ Yi Xu ${ }^{1} \cdot$ Zhijian Fang $^{1} \cdot$ Zhongyu Dai $^{1}$
}

Received: 18 January 2018/Revised: 26 January 2018 / Accepted: 7 February 2018/Published online: 5 March 2018

(C) Springer Science+Business Media, LLC, part of Springer Nature 2018

The Editor-in-Chief is retracting this article. After publication, the authors listed on the article informed the Editorin-Chief that they did not write the article. Attempts to contact the authors listed on the article via the contact details supplied to the journal on submission have failed to elicit any responses. Due to uncertainty around who contributed to and is accountable for this research, the Editorin-Chief has decided to retract this article.

Electronic supplementary material The online version of this article (https://doi.org/10.1007/s10586-018-2059-5) contains supplementary material, which is available to authorized users.

Junhua Wang

Purita_Herchell670@hotmail.com

Haoli Hou

Houhaoli@hotmail.com

Yi Xu

Zhaoji@hotmail.com

Zhijian Fang

BaNY@163.com

Zhongyu Dai

jIUSHIm@hotmail.com

1 School of Electrical Engineering, Wuhan University, Wuhan,

China 